\title{
Resolution Improvement Processing of Post-stack Seismic Data and Example Analysis - Taking NEB Gas Field As an Example in Indonesia
}

\author{
Chunlei Li ${ }^{1, a}$, Wenqi Zhang ${ }^{2, b}$, Chaohui Xia ${ }^{3, c}$, Ming Zhang ${ }^{4, d}$,Wei Ding ${ }^{5, e}$ \\ ${ }^{1}$ CNPC Research Institute of Petroleum Exploration \& Development (RIPED), China \\ ${ }^{2}$ CNPC Research Institute of Petroleum Exploration \& Development (RIPED), China \\ ${ }^{3}$ CNPC Research Institute of Petroleum Exploration \& Development (RIPED), China \\ ${ }^{4}$ CNPC Research Institute of Petroleum Exploration \& Development (RIPED), China \\ ${ }^{5}$ CNPC Research Institute of Petroleum Exploration \& Development (RIPED), China \\ alichunlei@petrochina.com.cn, bzhang_wenqi@petrochina.com.cn, cxiazhui@petrochina.com.cn, \\ dzhmlv@petrochina.com.cn, edngwei-hw@petrochina.com.cn,
}

Keywords: spectral inversion; resolution improvement; seismic data processing; constrained least square spectrum analysis (CLSSA).

Abstract. with continuous advance in reservoir prediction technology, the requirements for inversion accuracy are higher. It requires that seismic data must have features such as high fidelity, high SNR and high resolution. How to ensure the improvement of seismic data resolution on the premise of not reducing fidelity and SNR based on the existing seismic data is a difficult problem with which all geophysical engineers need to be confronted. In this paper, taking NEB gas field in Indonesia as an example, seismic data resolution is improved using the spectral inversion technology, the distribution characteristics of structures and faults in NEB oil and gas field are better ascertained, and the transverse connection of reservoirs and the distribution regularity of sand bodies are finely described, thus having great guidance significance to next development of NEB gas field.

\section{Introduction}

Spectral inversion (sparse layer inversion by spectrum restoration) is a high resolution seismic processing method of improving thin layer imaging precision with the spectral decomposition technology. The main principle of spectral inversion: partial spectrum information is obtained using the seismic frequency division method, multi-channel operators are determined through fine well-seismic calibration, then the reflection coefficient is calculated using the global optimization method and the high frequency components of original data are calculated trace by trace, and finally the obtained high frequency components are added back into original data, thereby obtaining the seismic data with higher resolution. Because of getting rid of man-made interference caused by well constraints, this inversion technology reflects the underground geologic features and reservoir features expressed by seismic data more truly and makes it possible to accurately predict inter-well thin reservoirs and precisely depict reservoirs. Through seismic resolution improvement processing, structural interpretation, reservoir prediction, fracture detection, hydrocarbon detection, etc. are finer and more accurate; in addition, the cost of seismic data acquisition and processing is reduced, and the existing seismic data can be utilized more fully.

\section{Spectral inversion processing technology for improving seismic resolution}

The research of predecessors indicate that peak amplitude is affected mainly by the lithology and physical properties of formations and fluid properties while peak frequency is affected mainly by formation thickness. In case of finding the characteristic frequency of formations with different thicknesses and then performing resolution improvement processing based on this, the problem on identification of thin reservoirs can be solved. 

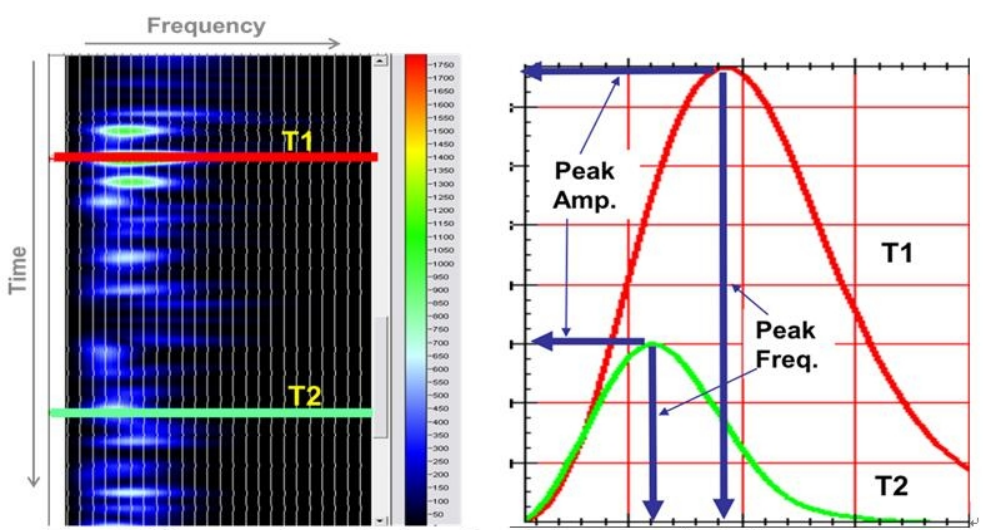

Fig. 1 Different peak amplitude and peak frequency (main frequency) responses

For example, the two sedimentary events $\mathrm{T} 1$ and $\mathrm{T} 2$ have different peak amplitude and peak frequency (main frequency) responses (Fig. 1). If not considering amplitude variation, the characteristic frequency (main frequency) is affected mainly by formation thickness variation. According to the spectral analysis, the characteristic frequency of $50 \mathrm{~ms}$ and $20 \mathrm{~ms}$ thick layers is respectively corresponding with $10 \mathrm{~Hz}$ and $25 \mathrm{~Hz}$, which are within the valid bandwidth, so thick layers can be identified directly. The characteristic frequency of $10 \mathrm{~ms}$ and $5 \mathrm{~ms}$ thin layers is respectively corresponding with $50 \mathrm{~Hz}$ and $100 \mathrm{~Hz}$, and the seismic data resolution capacity is not enough to identify thin layers. But the information on thin layers is implied in the valid bandwidth, and the spectrum restoration method can be used to expand bandwidth and improve data resolution (Fig. 2).

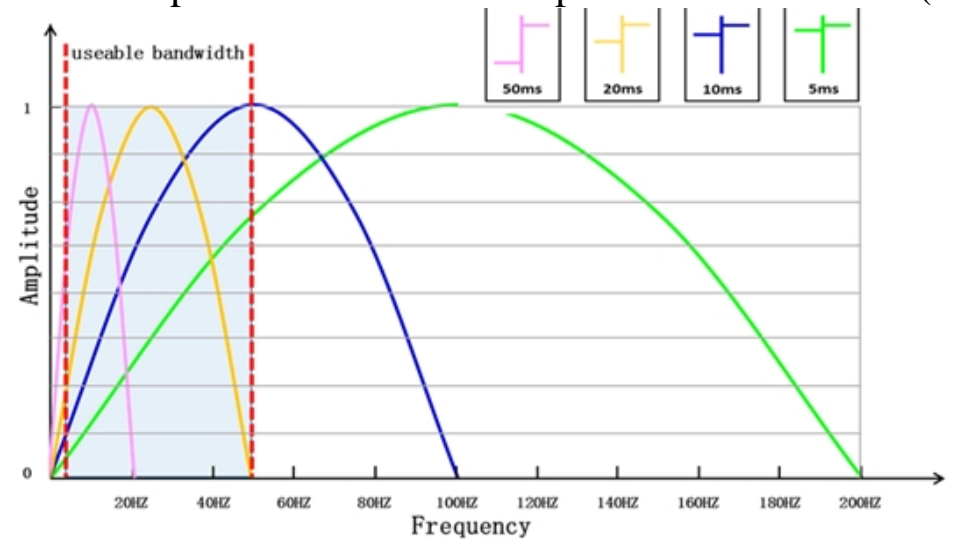

Fig. 2 Spectral view of different formation thicknesses

If the traditional seismic interpretation work is performed in the frequency domain which changed from the time domain, the accuracy and reliability are far too higher than those from conventional data interpretation can be obtained. A time domain single seismic trace is processed into frequency domain time-frequency gather display through spectral decomposition, and then it can be seen that similar different geologic events in the time domain have different characteristic frequency responses in the frequency domain. The method of converting seismic from time domain to frequency domain is spectral decomposition.

The traditional spectral decomposition algorithms include CLSSA (constrained least-squares method) and STFT (short-time Fourier transform). By comparing the two algorithms, it can be seen that the solution of spectrum obtained from CLSSA has better consistency with the theoretical spectrum, more abundant details and higher resolution and CLSSA has tremendous advantages in identification of thin layers, so CLSSA has been adopted in this study (Fig. 3). 


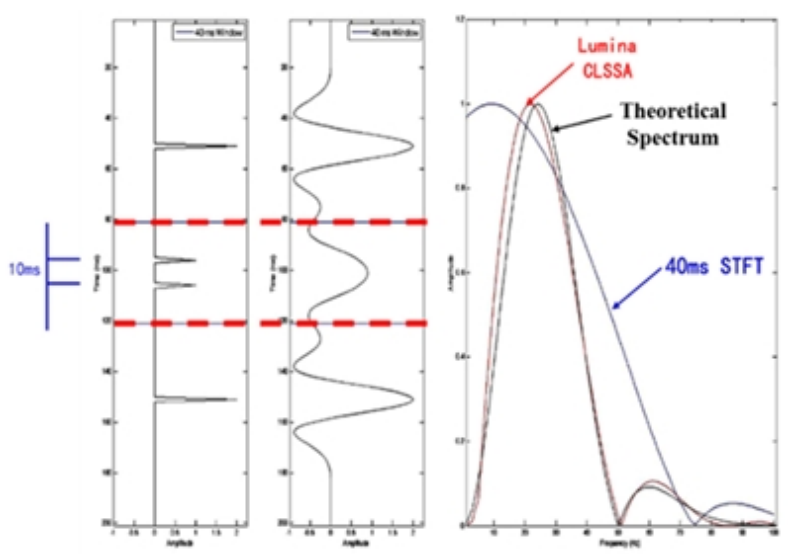

Fig. 3 Comparison of CLSSA and STFT methods

\section{Application example and effect analysis}

NEB gas field in Indonesia has very complex geologic features. In the gas field, multi-stage faults are developed in the plane, there are multiple sets of thin sandstone reservoirs longitudinally, fluid characteristics are very complex, and there are multiple oil, gas and water systems. After 10 years of high speed development, the complexity of structures, reservoirs and distribution of oil, gas and water in NEB gas field have been increasingly emerging. Taking NEB gas field as an example, seismic data resolution is improved with the spectral inversion technology through comprehensive analyses in this study, thus achieving the effect of finely depicting thin reservoirs.

\section{Data feasibility analysis before processing}

Firstly the feasibility of resolution improvement processing is analyzed through spectrum scanning of original seismic data. According to multi-band scanning and comparative analysis of original seismic data, $50-60 \mathrm{~Hz}$ data volumes imply reservoir detailed information, so it is feasible to carry out resolution improvement processing with this set of data volumes. The thin layer information implied in seismic data is restored with the resolution improvement processing technology to strive for the maximum useable bandwidth, and thus the processed high resolution data volumes can effectively support the geologic study and analysis of reservoirs (Fig. 4).

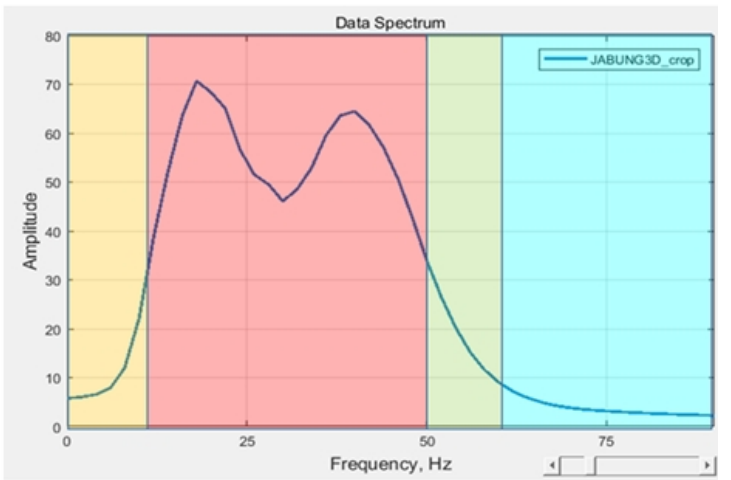

Fig. 4 Comparative analysis of seismic data within different bandwidth ranges

\section{Optimization processing with preserved amplitude and fidelity}

The main purpose of optimization processing with preserved amplitude and fidelity is to eliminate random noises, increase data SNR and provide high quality basic data for high resolution processing. According to the spectrum comparison before and after optimization, the spectrum characteristics are basically consistent; after processing, high-frequency invalid trailing data have been removed so as to avoid interference with post-processing (Fig. 5). 

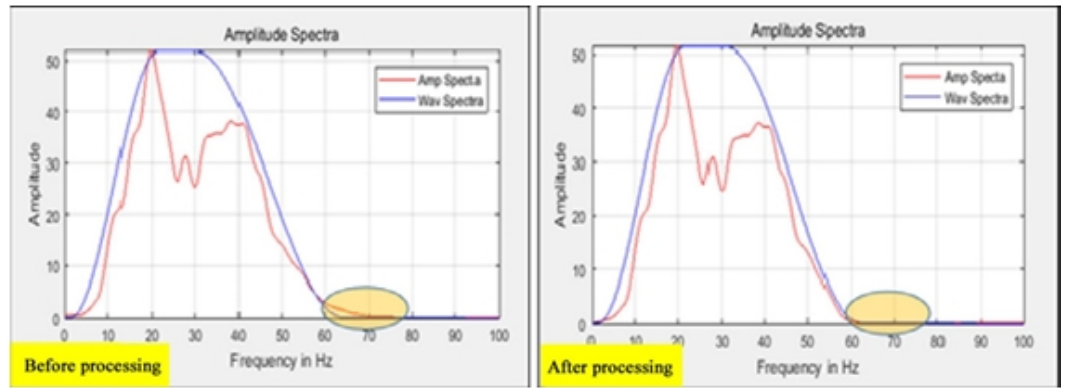

Fig. 5 Spectrum comparison before and after processing

Seismic resolution improvement processing by spectral inversion

The main purpose is to test parameters, optimize the best parameter combination and solve geologic problems. According to the comparative analysis of spectrum before and after processing, the main frequency of the target formation is $25 \mathrm{HZ}$ before processing and reaches $35 \mathrm{HZ}$ after processing, thus achieving the purpose of increasing the main frequency of the target formation and enlarging the bandwidth. The main frequency of the high frequency components has been increased from $55 \mathrm{HZ}$ to 75HZ (Fig. 6).
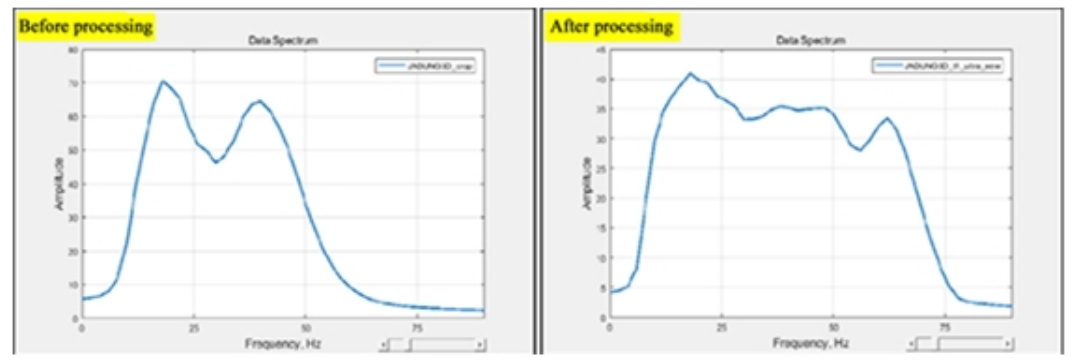

Fig. 6 comparison before and after seismic resolution improvement processing

\section{Imaging optimization processing}

The methods such as structure filtering etc. are mainly used to eliminate random noises and high frequency component interference and improve reservoir imaging quality, thus increasing seismic data interpretation accuracy and establishing a good foundation for next reservoir prediction and hydrocarbon detection (Fig. 7).
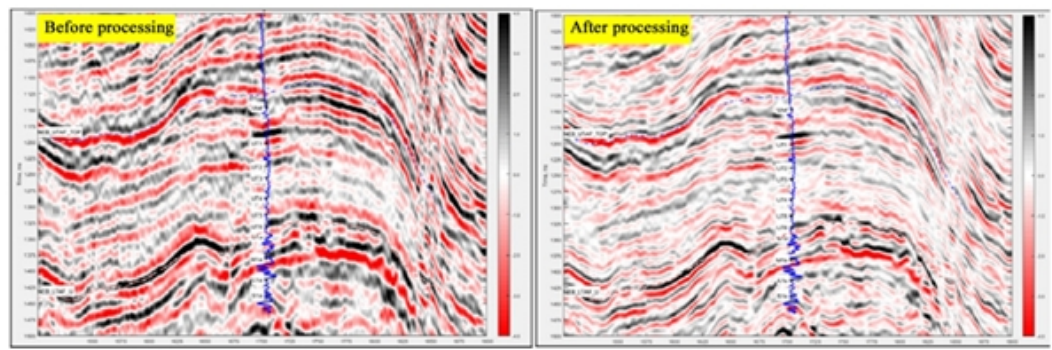

Fig. 7 Profile comparison before and after imaging optimization processing

\section{Analysis of data feasibility after resolution improvement processing}

After resolution improvement processing by spectral decomposition, the reliability of data can be analyzed by using multiple means, mainly including interlayer statistical attribute analysis, multi-seismic attribute analysis (profile, slice), fine well-seismic calibration analysis, etc.

a. interlayer statistical attribute analysis: according to the comparison of RMS amplitudes extracted from data volumes along the target interval before and after processing, they are very similar and the spatial relative amplitude relation is kept preferably, indicating high fidelity(Fig. 8). 


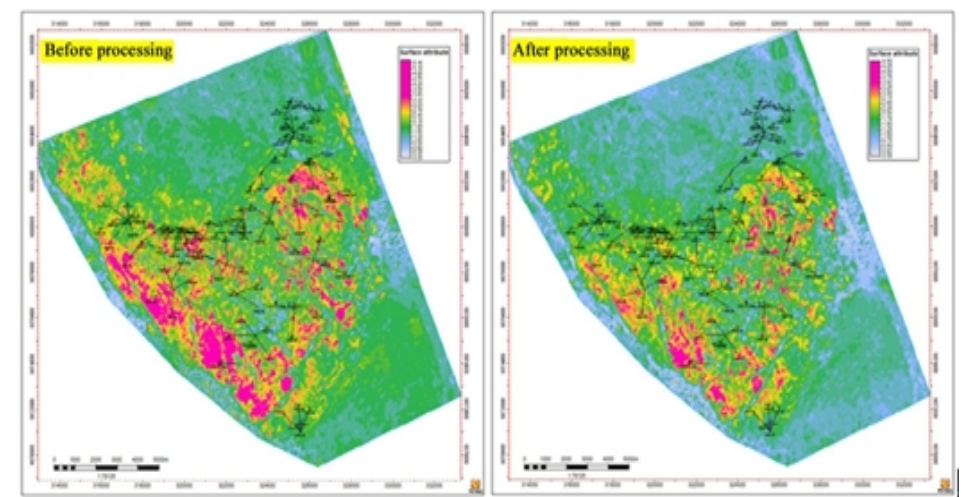

Fig. 8 Comparison of RMS amplitude attributes before and after processing

b. Multi-seismic attribute analysis (profile, slice): in order for more obvious effect comparison, the reliability of data after high resolution processing is verified through multi-seismic attribute comparison. According to the comparative analysis, it can be seen that the amplitude and phase relation keep consistent before and after processing and that the capacity of identifying the reservoirs in the target interval has been increased obviously (Fig. 9 and Fig. 10).

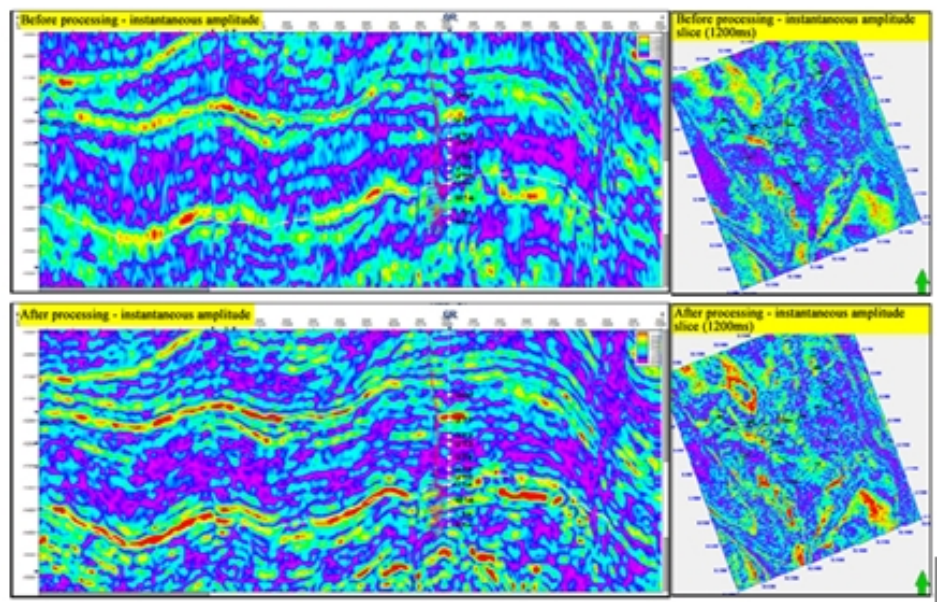

Fig. 9 Comparison of instantaneous amplitude attribute before and after processing

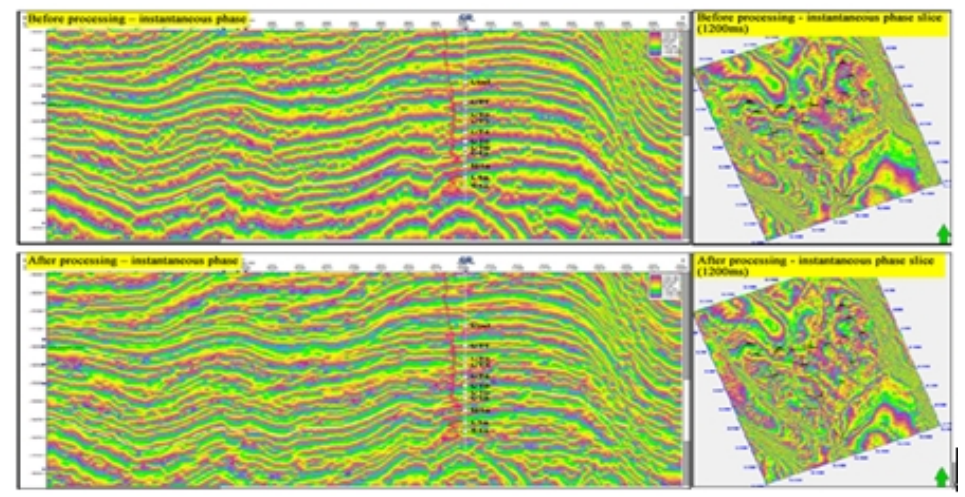

Fig. 10 Comparison of instantaneous phase attribute before and after processing

c. Fine well-seismic calibration: the effectiveness and reasonableness of resolution improvement processing are verified through fine well-seismic calibration. According to the comparison, it can be seen that the wave group relation before and after processing keeps basically consistent and that the capacity of identifying the reservoirs in the target interval has been increased obviously (Fig. 11). 

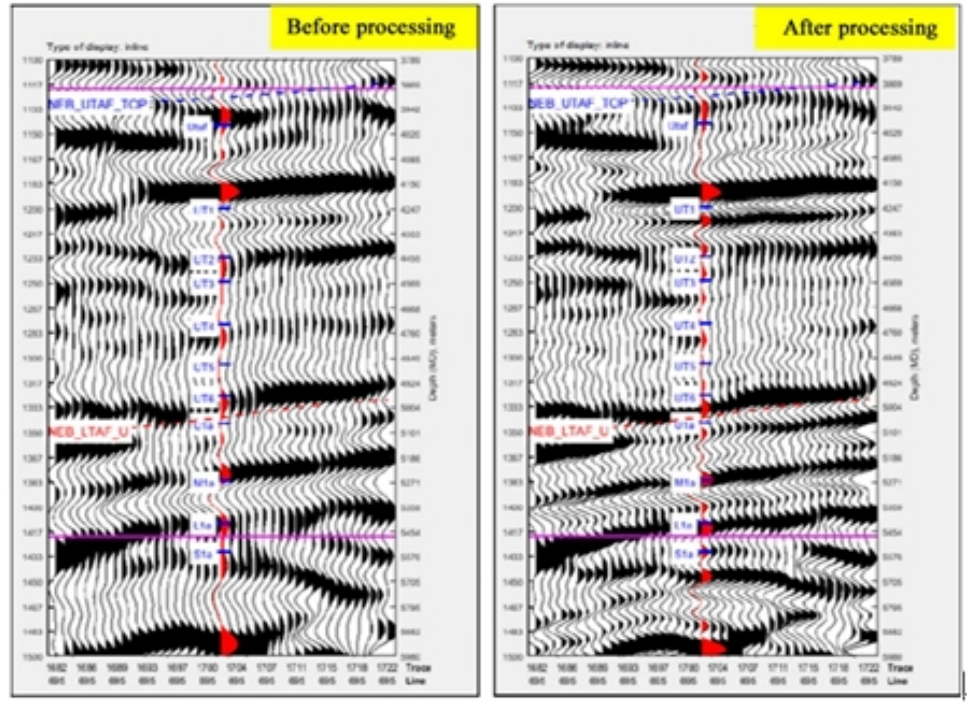

Fig. 11 Well-seismic calibration comparison before and after processing

\section{Conclusions}

After processing, the main frequency and bandwidth of seismic data have been increased and seismic resolution has been improved remarkably. The spectral inversion technology is based on preservation of fidelity and amplitude and thus fully ensures data reliability and the validity of high frequency components. The spectral inversion technology has a reliable quality control and analysis process, plays a key role in effectively improving seismic resolution and greatly increases the capacity of seismic identification of thin layers, thereby establishing a substantial foundation for next fine reservoir prediction and hydrocarbon detection.

\section{References}

[1] Wang Zhuwen, Wang Xiaoli, Xiang Min, Liu Jinghua, Zhang Xueang, Yang Chuang. Reservoir information extraction using a fractional Fourier transform and a smooth pseudo Wigner-Ville distribution (SPWD) (English) [J]. Applied Geophysics. 2012(04).

[2] Chen Xuehua, He Zhenhua, Huang Deji, Wen Xiaotao. Low frequency shadow detection of gas reservoirs in time-frequency domain [J]. CHINESE JOURNAL OF GEOPHYSICS. 2009(01).

[3] Han Li, Han Liguo. Reassignment seismic spectrum decomposition technology based on varying window parameter [A]. China Geophysics 2012[C]. 2012.

[4] M. Kulesh,M. Holschneider,M. S. Diallo,Q. Xie,F. Scherbaum. Modeling of Wave Dispersion Using Continuous Wavelet Transforms[J]. Pure and Applied Geophysics . 2005 (5).

[5] Xiaoyang Wu,Tianyou Liu. Seismic spectral decomposition and analysis based on Wigner-Ville distribution for sandstone reservoir characterization in West Sichuan depression. Journal of Geophysics and Engineering . 2010

[6] Sinha S,Routh P S,Anno P D et al. Spectral decom-position of seismic data with continuous-wavelet transform. Geophysics. 2005.

[7] Buland A,Kolbjornsen O,Omre H. Rapid spatiallycoupled AVOinversion in the Fourier domain. Geophysics. 2003. 